\title{
Novel homozygous HEXB mutation identified in a consanguineous Iranian pedigree with Sandhoff disease
}

\section{Zahra Rahmani}

Golestan University of Medical Sciences and Health Services

\section{Arsham Banisadr}

Mashhad University of Medical Sciences

Vadieh Ghodsinezhad

Tehran University of Medical Sciences

Mohsen Dibaj

University of Tabriz

Omid Aryani ( $\square$ o_aryani@yahoo.com )

Iran University of Medical Sciences

\section{Primary research}

Keywords: HEXB, Sandhoff disease, Mutation

Posted Date: January 23rd, 2020

DOl: https://doi.org/10.21203/rs.2.21720/v1

License: (c) (1) This work is licensed under a Creative Commons Attribution 4.0 International License. Read Full License 


\section{Abstract}

Background Sandhoff disease is a rare neurodegenerative and autosomal recessive disorder, characterized by a defect in ganglioside metabolism. It is caused by mutations in the HEXB gene for the $\beta$-subunit of $\beta$ - $\mathrm{N}$-acetyl hexosaminidase. Results In the present study, an Iranian 14- month -old girl with an 8- month history of unsteady walking and involuntary movements is described. Biochemical testing showed defects in the normal activity of beta-hexosaminidase protein. Following sequencing of HEXB gene, a novel homozygous p.A278V mutation was identified in the patient's DNA. Conclusions The p.A278V mutation is pathogenic because of amino acid change and changing in biochemical activity. this mutation has not been reported previously, but based on In silico analysis and structural analysis, was predicted to be disease causing.

\section{Introduction}

Sandhoff disease (SD, OMIM 268800), is an autosomal recessive neurodegenerative disorder that was initially described by Konrad Sandhoff in1968. It is occurred due to the mutation in the beta subunit of hexosaminidase [1] [2]. HEXA and HEXB are two major Hex isoenzymes. b-HEXA consisting of $a / \beta$ heterodimer subunits, whereas $b$-Hex $B$ is composed of $B / B$ homodimer subunits. Mutations affecting HEXB gene result in Sandhoff disease, so both HexA and HexB isoenzyme activities are decreased or absent [3]. The HEXB isoenzyme is a catalytic enzyme accountable for degrading the GM2 ganglioside[4]. HEXB gene which is about $40 \mathrm{~kb}$ long, contains 14 exons and located on chromosome $5 q 13$ [5]. The prevalence of Sandhoff disease is 1 in 384000 live births and many different pathogenic variants have been reported in patients with Sandhoff disease in the Human Gene Mutation Database (HGMD at http://www.hgmd.org/). They consist of 43 missense/nonsense, 3small insertions, 19 small deletions, 6 gross deletions and 18 splicing mutation [5]. The most prevalent mutation is a $16 \mathrm{~kb}$ deletion at the 5 ' end of the gene. This deletion leads to loss of exon 1-5 and the HEXB promoter [6]. Some of them have been reported as the most common mutation in some populations. The c.171delG (p.W57CfsX6) deletion was found in accounting for $21.4 \%$ of the alleles in Spain and showed the infantile form of the disease [7]. Abnormality of HEXB results in a deficiency of hexosaminidase A and B that are responsible for lysosomal accumulation of GM2 gangliosides and linked glycolipids, mostly in neurons, describing neurological symptoms in this disease [8] [9]. Classic infantile, late infantile, juvenile and adult late onset are different forms of Sandhoff disease [2]. Each category has its feature in severity and age at the onset of disease [10]. There are several signs which may include Weakness, blindness, progressive mental and motor deterioration, macrocephaly [11].The infantile form of Sandhoff disease is manifested by the early onset of signs, which usually occurs within the first 6 months of life[10]. In the classic form of Sandhoff disease, hexosaminidase activity is absent [12]. The juvenile form of Sandhoff disease indicates symptoms between the ages of 4 and 6 years [13]. Symptoms include ataxia, motor skills regression, learning disorders and some autistic features [14]. The adult form of Sandhoff disease usually is characterized by neurologic symptoms in early childhood [15]. The disorder in adult-onset patients 
affects, mainly the motor neuron, cerebellar and autonomic functions [16]. In current work we are going to evaluate that weather this mutation has any effect on the function of HEX B or not.

\section{Results}

\section{Clinical finding}

The clinical feature of child at first presentation was developmental delay with a mean age at presentation of 4 months. Cognitive decline was attained after 9 months of age. After 10 months of age, there was regression in speech. The child complained of seizure, truncal hypotonia and lower limbs spasticity. Physical examination and then ultrasonography revealed no splenomegaly and hepatomegaly. Ophthalmologic examination revealed cherry red spots in both macular areas in patient. The lysosomal enzymatic activities indicated reduction of $\beta$-hexosaminidase B (HEXB) activity. A novel homozygous p.A278V mutation was identified in the patient. Brain magnetic resonance imaging (MRI) indicated signal changes over the bilateral thalami, bilateral cerebral white matter and left putamen.

\section{Biochemical enzyme assay}

The biochemical enzyme assay showed a deficiency of both hexosaminidases $A(0.19 \mathrm{nmol} / \mathrm{ml} / \mathrm{min})$ and $\mathrm{B}(0.08 \mathrm{nmol} / \mathrm{ml} / \mathrm{min})$, compatible with a diagnosis of Sandhoff disease (Table 1$)$.

We identified a novel c.833C>T (p.A278V) homozygous mutation in exon 7 of the HEXB gene. Both parents were heterozygous for this mutation (Fig.1).

To predict the possible effects of the new mutation on the structure and function of HEXB protein, the p.A278V mutation was analyzed using SIFT and PolyPhen (Table 2). Here, SIFT result indicated that p.A278V mutation was predicted as deleterious, with SIFT scores of -3.735. Based on the PolyPhen score, the p.A278V mutation was found as "Probably Damaging" to protein structure and function, with a score of 1.000 . The structure of the region around Ala 278 was shown in Fig.2, using the PyMOL molecular graphics program (14).

Multiple-sequence alignment of seven HEXB homologs (human, chimpanzee, rhesus monkey, cow, dog, rat, chicken), using ClustalOmega revealed that codon 278 was located within a highly conserved region (Fig.3).

We compared mutant and wild protein structures, using YASARA software, difference of 3D structure was $0.5434 \AA$. The interaction energy of mutant protein to GDL $(-0.09 \mathrm{kcal} / \mathrm{mol})$ (Table 3) (Figure 4), was obviously lower than wild protein to GDL $(-196.35 \mathrm{kcal} / \mathrm{mol})$. We also compared interaction energy of wild and mutant protein to NAG, EDO (Table 3).

\section{Discussion}


Sandhoff disease is a rare metabolic disease and results from unusual accumulation of gangliosides leads to progressive deterioration of the central nervous system. Sandhoff disease individuals have missing or decreased activity of beta-hexosaminidase A and beta-hexosaminidase B [29].It can be detected by making a lysosomal enzyme assay to show activity of the hexosaminidase $A$ and $B$ enzymes [30].

There is genetic testing for determination of specific pathogenic mutations that is existent in the beta subunit of hexosaminidase (HEXB) to corroborate the diagnosis, on the HEXB mutations.

To date, some Sandhoff disease in Iranian population with pathogenic variants have been reported.

For instance, $\mathrm{H}$ Aryan et al, have reported 7 various mutations including c.1552delG, c.410G>A, c.362 A>G, c.550delT, c. $1597 \mathrm{C}>\mathrm{T}, \mathrm{c} .1752 \mathrm{delTG}$ and the most common findings (50\%) was a $16 \mathrm{~kb}$ deletion including the promoter and exons 1-5. This mutation was not observed in healthy controls [10].

Other studies have identified pathogenic missense mutation c.821T N A (p.Val274Glu; Homozygous) in exon 7 of the HEXB gene, lead to unusual enzyme activity [5].

Also, some mutation reported from various geographic areas and population such as South Americans, Cyprus, Italians, Middle East Arabs, Spanish, French, Thailand, and Korean.

By the way, Wang et al have reported a homozygous missense HEXB mutation (p. D459A) in six patients with a rare juvenile variant [31]. Additionally, a new c.1556A>G transition in exon 12 of the HEXB gene has recognized, covert aspartic acid to glycine at the position 494 of the Hex B-subunit, correlated with Sandhoff disease [32]. Later, Stefania Zampieri identified 9 novel sequence variants in patients with Sandhoff disease with different geographical/ethnic background including 4 sequence variations leading to residue sequence changes [c.626C.T (p.T209I), c.634C.A (p.H212N), c.926G.T (p.C309F), c.1451G.A (p.G484E)] 3 intronic mutations (c.1082+5G.A, c.1242+1G.A, c.1169+5G.A), 1 nonsense mutation c.146C.A (p.S49X) and 1 small in-frame deletion c.1260_1265de-IAGTTGA (p.V421_E422del) [3]. Furthermore, Gomez-Lira et al reported a novel 4-bp deletion in exon 4 infantile-onse Sandhoff disease that was the explanation for the disease [33].

Seven novel mutations on the HEXB gene in French Sandhoff patients were recognized and their pathogenicity was confirmed by in silico analysis [8]. Before that, Macarena Gomez-Lira et al identified a novel mutation in the HEXB gene, a 48-bp Insertion Between Exon 13 and 14, in infantile-Onset Sandhoff Disease [34]. As well, Kenichiro Yamada et al have reported compound heterozygous mutations, $\mathrm{H} 235 \mathrm{Y}$ and P417L, in HEXB which were linked with the adult form of Sandhoff disease in 46-year-old Japanese male, The patient's parents were non-consanguineous, with no history of motor neuron disease in any family members [35]. Four missense mutations including Y456S, P504S, R533H and P417L correlated with the adult form of Sandhoff disease with the motor neuron disease phenotype have been recognized [36][16][37][38][39]. 


\section{Conclusions}

Our study identifies a new case of Sandhoff disease with new mutation is Characterized.

Our results showed that mutant protein is pathogenic because of the amino acid alteration (p.A278V) and biochemical evaluation confirmed low b-hexosaminidase A and B activities. Moreover, structural and Molecular docking analysis revealed variability in mutant HEX B. The interaction energy of protein-ligand was obtained, using Hex 8.0.0 software. And the docking results showed, GDL interacts more strongly with native protein in comparison to mutant.

GDL acts as Protein homodimerization [40][41].

Several attempts have been made toward treatment including pharmacological chaperones [42], substrate reduction with or without gene therapy [43].

Gene therapy may be a hopeful treatment modality, but is unlikely to be available for years. Given the magnitude of carrier burden in recessive diseases and the lower cost of diagnostic genetic tests compared with treating, diagnostic screening of family members may be useful.

\section{Methods}

\section{Case Report}

A 14 months old girl was referred to Endocrinology and Metabolism Research Institute (EMRI/TUMS) with psychomotor regression from 7 months age. She was born after a full-term pregnancy with head circumference of $33 \mathrm{~cm}$ and birth weight of $3.200 \mathrm{Kg}$ from a consanguineous marriage (first cousin couple) and positive family history (the previous male child died at 18 months age with the same phenotype).

\section{$\beta$-Hexosaminidase activity assay}

The activity of $\beta$-Hexosaminidase was measured via dried blood spots (DBSs) on filter.

The blood samples from patient was collected and spotted on filter paper and placed in $3 \mathrm{~mm}$ diameter. After addition of elution liquid and substrate solution then incubate of the samples at $37^{\circ} \mathrm{C}$, the enzyme activity quantified by comparing the amount of hydrolyzed product with a calibrator [17] [18].

\section{Mutational Analysis of HEXB Gene}

Genomic DNA of the patient was extracted from peripheral blood leukocytes according to the standard procedure using FlexiGene DNA Kit (QIAGEN). The exonic and flanking intronic sequences of the HEXB gene were amplified by PCR and examined for specificity via 1.5\% agarose gel electrophoresis. The PCR products were sequenced on an $\mathrm{ABI} 3700$ sequencer (Kosar Company, Tehran) and compared with the wild-type HEXB sequence (NM_000521.3). 


\section{In Silico Analysis}

To predict the possible effects of the new mutation on the structure and function of HEXB protein, the sequence alteration were assessed by the in silico prediction algorithms SIFT and Polyphen-2 [19] [20].

\section{Structural Analysis}

The structures of Beta-hexosaminidase subunit beta was carried out using molecular modeling tools server [21].the sequences and 3D shapes of hex $b$ as well as ligand were acquired from protein databank (PDB) [22].The crystallographic structure of molecules was removed for the existence of water atoms and other unnecessary additional chains using UCSF Chimera 1.8.1 [23][24]. Protein homology modeling of the newly-identified mutated HEXB protein, $\mathrm{p} . \mathrm{A} 278 \mathrm{~V}$, was carried out using the online Swiss-Prot server for automated modeling [25].protein structure prediction server, RaptorX, was also used for homology based 3D structure prediction [26].

x-ray crystallography was obtained to better understand the structural effects caused by mutations in Beta-hexosaminidase subunit beta (Accession code: 107A) [27].protein-ligand docking were calculated from Hex 8.0.0 software. We used Hex 8.0.0 in docking of 2-acetamido-2-deoxy-d-glucono-1,5-lactone (GDL), n-acetyl-d-lucosamine (NAG), 1,2-ethanediol (EDO) to the target protein hex b structure( wild and mutant type) [28][29][27]. the ligand binding energy of these complexes also were assessed.

\section{List Of Abbreviations}

Hexosaminidases B (HEX B)

Hexosaminidases A (HEX A)

2-acetamido-2-deoxy-d-glucono-1,5-lactone (GDL)

n-acetyl-d-lucosamine (NAG)

1,2-ethanediol (EDO)

\section{Declarations}

Ethics approval and consent to participate

Not applicable

Consent for publication

Not applicable

Availability of data and materials 
The activity of $\beta$-Hexosaminidase was measured via dried blood spots (DBSs) on filter.

Genomic DNA extraction using FlexiGene DNA Kit (QIAGEN).

Sequencing on an ABI 3700 sequencer (Kosar Company, Tehran).

The sequence alteration was assessed by the in silico prediction algorithms SIFT and Polyphen-2.

The structures of Beta-hexosaminidase subunit beta using UCSF Chimera 1.8.1, online Swiss-Prot server, RaptorX.

Protein-ligand docking was calculated from Hex 8.0.0 software.

\section{Competing interests}

The authors declare that they have no competing interests

\section{Funding}

No funding received

\section{Authors' contributions}

All authors read and approved the final manuscript

\section{Acknowledgements}

We thank the parent s patient and also thank all physicians who supported us via examination, provision of blood samples and etc.

\section{Authors' information}

First Author: Zahra Rahmani

Department of Medical Genetics, Golestan University of Medical Sciences, Gorgan, Iran.

Second Author: Arsham Banisadr

Department of Medical Biotechnology and Nanotechnology/ Mashhad university of Medical Sciences, Mashhad, Iran.

Third Author: Vadieh Ghodsinezhad

Medical Genetics Center, Endocrinology and Metabolism Research Institue (EMRI), Tehran University of Medical Sciences (TUMS), Tehran, Iran.

Fourth $\llbracket$ Author: Mohsen Dibaj 
Molecular Genetic Department, University of Tabriz, Tabriz, Iran.

Corresponding Author: Omid Aryani

Neuroscience Department, Iran University of Medical sciences, Tehran, Iran.

\section{References}

[1] B. Boonyawat et al., "HHS Public Access," Iran. J. Public Health, vol. 21, no. 1, pp. 281-285, 2015.

[2] B. Boonyawat, T. Phetthong, C. Nabangchang, and P. Suwanpakdee, "A novel frameshift mutation of HEXA gene in the first family with classical infantile Tay-Sachs disease in Thailand," Neurol. Asia, vol. 21, no. 3, pp. 281-285, 2016.

[3] S. Zampieri et al., "Sequence and Copy Number Analyses of HEXB Gene in Patients Affected by Sandhoff Disease: Functional Characterization of 9 Novel Sequence Variants," vol. 7, no. 7, pp. 1-10, 2012.

[4] W. Zhang, H. Zeng, Y. Huang, T. Xie, and J. Zheng, "Clinical, biochemical and molecular analysis of five Chinese patients with Sandhoff disease," 2016.

[5] R. Ebrahimzadeh-Vesal, S. Hosseini, M. Moghaddassian, and M. R. Abbaszadegan, "Identification of novel missense HEXB gene mutation in Iranian-child with juvenile Sandhoff disease," Meta Gene, vol. 12, pp. 83-87, 2017.

[6] K. Neote, B. Mcinnes, D. J. Mahuran, and R. A. Gravel, "Structure and Distribution of an Alu-type Deletion Mutation in Sandhoff Disease."

[7] L. Gort, N. De Olano, J. Macías-vidal, and M. Josep, "GM2 gangliosidoses in Spain: Analysis of the HEXA and HEXB genes in 34 Tay - Sachs and 14 Sandhoff patients $\otimes$, , Gene, vol. 506, no. 1, pp. 25-30, 2012.

[8] P. Gaignard et al., "Characterization of seven novel mutations on the HEXB gene in French Sandhoff patients," Gene, vol. 512, no. 2, pp. 521-526, 2013.

[9] S. Lakshmi, G. Anitha, and S. Vinoth, "A rare case of Sandhoff disease: two in the same family," Int. J. Contemp. Pediatr., vol. 2, no. 1, p. 42, 2015.

[10] H. Aryan, O. Aryani, K. Banihashemi, T. Zaman, and M. Houshmand, "Novel mutations in sandhoff disease: A molecular analysis among Iranian cohort of infantile patients," Iran. J. Public Health, vol. 41, no. 3, pp. 112-118, 2012.

[11] M. Abiri, S. Talebi, J. Uitto, L. Youssefian, H. Vahidnezhad, and T. Shirzad, “Co-existence of phenylketonuria either with maple syrup urine disease or Sandhoff disease in two patients from Iran: 
emphasizing the role of consanguinity," 2016.

[12] K. Sandhoff, H. Christomanou, M. Psychiatrie, and N. Abteilung, "Biochemistry and Genetics of Gangliosidoses," vol. 143, pp. 107-108, 1979.

[13] T. N. and K. Suzuki, "Genetic Cause of a Juvenile Form of Sandhoff Disease," DNA Seq., vol. 264, no. 9 , pp. 5155-5158, 1989.

[14] W. Biochemical, G. Unit, and C. D. Centre, "Juvenile Sandho ¡ diseaseöNine new cases and a review of the literature," vol. 27, pp. 241-249, 2004.

[15] P. A. Bolhuis, N. J. Ponne, H. Bikker, F. Baas, and J. M. B. V. de Jong, "Molecular basis of an adult form of Sandhoff disease: Substitution of glutamine for arginine at position 505 of the??-chain of ??hexosaminidase results in a labile enzyme," BBA - Mol. Basis Dis., vol. 1182, no. 2, pp. 142-146, 1993.

[16] M. G. A. Sangalli, M. Mottes, C. Perusi, P. Franco, N. Rizzuto, and A. Salviati, "A common hexosaminidase gene mutation in adult Sandhoff disease patients," pp. 417-418, 1995.

[17] N. A. Chamoles, M. Blanco, D. Gaggioli, and C. Casentini, "Tay-Sachs and Sandhoff diseases: Enzymatic diagnosis in dried blood spots on filter paper: Retrospective diagnoses in newborn-screening cards," Clin. Chim. Acta, vol. 318, no. 1-2, pp. 133-137, 2002.

[18] A. R. Tavasoli, N. Parvaneh, M. R. Ashrafi, Z. Rezaei, J. Zschocke, and P. Rostami, "Clinical presentation and outcome in infantile Sandhoff disease: A case series of 25 patients from Iranian neurometabolic bioregistry with five novel mutations Dr. Segolene Ayme," Orphanet J. Rare Dis., vol. 13, no. 1, pp. 1-8, 2018.

[19] I. A. Adzhubei et al., "A method and server for predicting damaging missense mutations a," Nat. Publ. Gr., vol. 7, no. 4, pp. 248-249, 2010.

[20] P. Kumar, S. Henikoff, and P. C. Ng, "Predicting the effects of coding non-synonymous variants on protein function using the SIFT algorithm," vol. 4, no. 8, pp. 1073-1082, 2009.

[21] L. A. Kelly, S. Mezulis, C. Yates, M. Wass, and M. Sternberg, "The Phyre2 web portal for protein modelling, prediction, and analysis," Nat. Protoc., vol. 10, no. 6, pp. 845-858, 2015.

[22] H. M. Berman et al., "The protein data bank.," Nucleic Acids Res., vol. 28, no. 1, pp. 235-242, 2000.

[23] E. F. Pettersen et al., "UCSF Chimera - A visualization system for exploratory research and analysis," J. Comput. Chem., vol. 25, no. 13, pp. 1605-1612, 2004.

[24] A. Mohebbi, S. Mohammadi, and A. Memarian, "Prediction of HBF-0259 interactions with hepatitis B Virus receptors and surface antigen secretory factors," VirusDisease, vol. 27, no. 3, pp. 234-241, 2016. 
[25] R. Apweiler et al., "The Universal Protein resource (UniProt) 2009," Nucleic Acids Res., vol. 37, no. SUPPL. 1, pp. 169-174, 2009.

[26] E. a Akl et al., "Parenteral anticoagulation may prolong the survival of patients with limited small cell lung cancer: a Cochrane systematic review," Proteins Struct. Funct. Bioinforma., vol. 10, no. SUPPL. 10, pp. 1-10, 2008.

[27] T. Maier, N. Strater, C. G. Schuette, R. Klingenstein, K. Sandhoff, and W. Saenger, "The X-ray crystal structure of human $\beta$-hexosaminidase B provides new insights into Sandhoff disease," J. Mol. Biol., vol. 328 , no. 3, pp. 669-681, 2003.

[28] E. M. Leslie, R. G. Deeley, and S. P. C. Cole, "Multidrug resistance proteins: Role of P-glycoprotein, MRP1, MRP2, and BCRP (ABCG2) in tissue defense," Toxicol. Appl. Pharmacol., vol. 204, no. 3, pp. 216237, 2005.

[29] Y. Hou, D. Vocadlo, S. Withers, and D. Mahuran, "Role of Arg 211 in the Active Site of Human Hexosaminidase B †," Society, vol. 39, no. 20, pp. 6219-6227, 2000.

[30] Y. Yun and S. Lee, "A case refort of Sandhoff disease.," Korean J. Ophthalmol., vol. 19, no. 1, pp. 68-72, 2005.

[31] S. Z. Wang et al., "A novel HEXB mutation and its structural effects in juvenile Sandhoff disease," Mol. Genet. Metab., vol. 95, no. 4, pp. 236-238, 2008.

[32] M. Santoro et al., "Chronic GM2 gangliosidosis type Sandhoff associated with a novel missense HEXB gene mutation causing a double pathogenic effect," Mol. Genet. Metab., vol. 91, no. 1, pp. 111-114, 2007.

[33] M. Gomez-Lira et al., "A novel 4-bp deletion creates a premature stop codon and dramatically decreases HEXB mRNA levels in a severe case of Sandhoff disease," Mol. Cell. Probes, vol. 15, no. 2, pp. 75-79, 2001.

[34] M. Gomez???Lira et al., "A 48???bp insertion between exon 13 and 14 of the HEXB gene causes infantile???onset sandhoff disease," Hum. Mutat., vol. 6, no. 3, pp. 260-262, 1995.

[35] K. Yamada, Y. Takado, Y. S. Kato, Y. Yamada, H. Ishiguro, and N. Wakamatsu, “Characterization of the mutant $\beta$-subunit of $\beta$-hexosaminidase for dimer formation responsible for the adult form of Sandhoff disease with the motor neuron disease phenotype," J. Biochem., vol. 153, no. 1, pp. 111-119, 2013.

[36] P. Banerjee et al., "Molecular basis of an adult form of beta-hexosaminidase B deficiency with motor neuron disease.," Biochem. Biophys. Res. Commun., vol. 181, no. 1, pp. 108-15, 1991. 
[37] T. Yoshizawa, Y. Kohno, S. Nissato, and S. Shoji, “Compound heterozygosity with two novel mutations in the HEXB gene produces adult Sandhoff disease presenting as a motor neuron disease phenotype," J. Neurol. Sci., vol. 195, no. 2, pp. 129-138, 2002.

[38] M. Rubin, G. Karpati, L. S. Wolfe, S. Carpenter, M. H. Klavins, and D. J. Mahuran, "Adult onset motor neuronopathy in the juvenile type of hexosaminidase A and B deficiency," J. Neurol. Sci., vol. 87, no. 1, pp. $103-119,1988$.

[39] Y. Hou, B. McInnes, A. Hinek, G. Karpati, and D. Mahuran, "A Pro504 $\rightarrow$ Ser substitution in the $\beta$ subunit of $\beta$-hexosaminidase $A$ inhibits a-subunit hydrolysis of $G(M 2)$ ganglioside, resulting in chronic Sandhoff disease," J. Biol. Chem., vol. 273, no. 33, pp. 21386-21392, 1998.

[40] J. P. Overington, B. Al-Lazikani, and A. L. Hopkins, "How many drug targets are there?," Nat. Rev. Drug Discov., vol. 5, no. 12, pp. 993-996, 2006.

[41] P. Imming, C. Sinning, and A. Meyer, "Drugs, their targets and the nature and number of drug targets," Nat. Rev. Drug Discov., vol. 5, no. 10, pp. 821-834, 2006.

[42] G. H. B. Maegawa et al., "Pyrimethamine as a potential pharmacological chaperone for late-onset forms of GM2 gangliosidosis," J. Biol. Chem., vol. 282, no. 12, pp. 9150-9161, 2007.

[43] M. B. Cachon-Gonzalez, S. Z. Wang, A. Lynch, R. Ziegler, S. H. Cheng, and T. M. Cox, "Effective gene therapy in an authentic model of Tay-Sachs-related diseases," Proc. Natl. Acad. Sci., vol. 103, no. 27, pp. 10373-10378, 2006.

\section{Tables}

Table 1: the biochemical enzyme assay

\begin{tabular}{|l|l|l|}
\hline Clinical chemistry & $\mathrm{nmol} / \mathrm{ml} / \mathrm{min}$ & Normal range \\
\hline Hexosaminidases A & 0.19 & $0.96-1.78$ \\
\hline Hexosaminidases AB & 0.99 & $18.59-31.33$ \\
\hline Hexosaminidases B & 0.08 & $5.76-15.77$ \\
\hline
\end{tabular}

Table 2: Bioinformatics Analysis of the novel mutation. 


\begin{tabular}{|l|l|l|l|l|l|}
\hline \multicolumn{2}{|c|}{} & \multicolumn{4}{c|}{ Bioinformatics Analysis } \\
\hline DNA level & Amino acid level & \multicolumn{2}{c|}{ PolyPhen-2 } & \multicolumn{2}{c|}{ SIFT } \\
\hline c.833C>T & $\begin{array}{l}\text { NM_000521.3 } \\
\text { p.A278V }\end{array}$ & Prediction & Score & Prediction & Score \\
\cline { 3 - 6 } & Probably Damaging & 1 & Deleterious & -3.735 \\
\hline
\end{tabular}

PolyPhen Prediction Score : Benign( < 0.5); Probably Damaging $(0.5<)$.

SIFT Prediction Score : Deleterious $(<0.05)$; Tolerated $(>0.05)$.

Table 3: Docking total energy of protein- ligand.

\begin{tabular}{|l|l|l|l|}
\hline & $\begin{array}{l}\text { Interaction Energy to NAG } \\
(\mathrm{Kcal} / \mathrm{mol})\end{array}$ & $\begin{array}{l}\text { Interaction Energy to } \\
\text { EDO (Kcal/mol) }\end{array}$ & $\begin{array}{l}\text { Interaction Energy } \\
\text { to GDL }\end{array}$ \\
\hline $\begin{array}{l}\text { Wild } \\
\text { protein }\end{array}$ & -218.77 & -101.43 & -196.35 \\
\hline $\begin{array}{l}\text { Mutant } \\
\text { protein }\end{array}$ & -192.28 & -98.30 & -0.09 \\
\hline
\end{tabular}

\section{Figures}

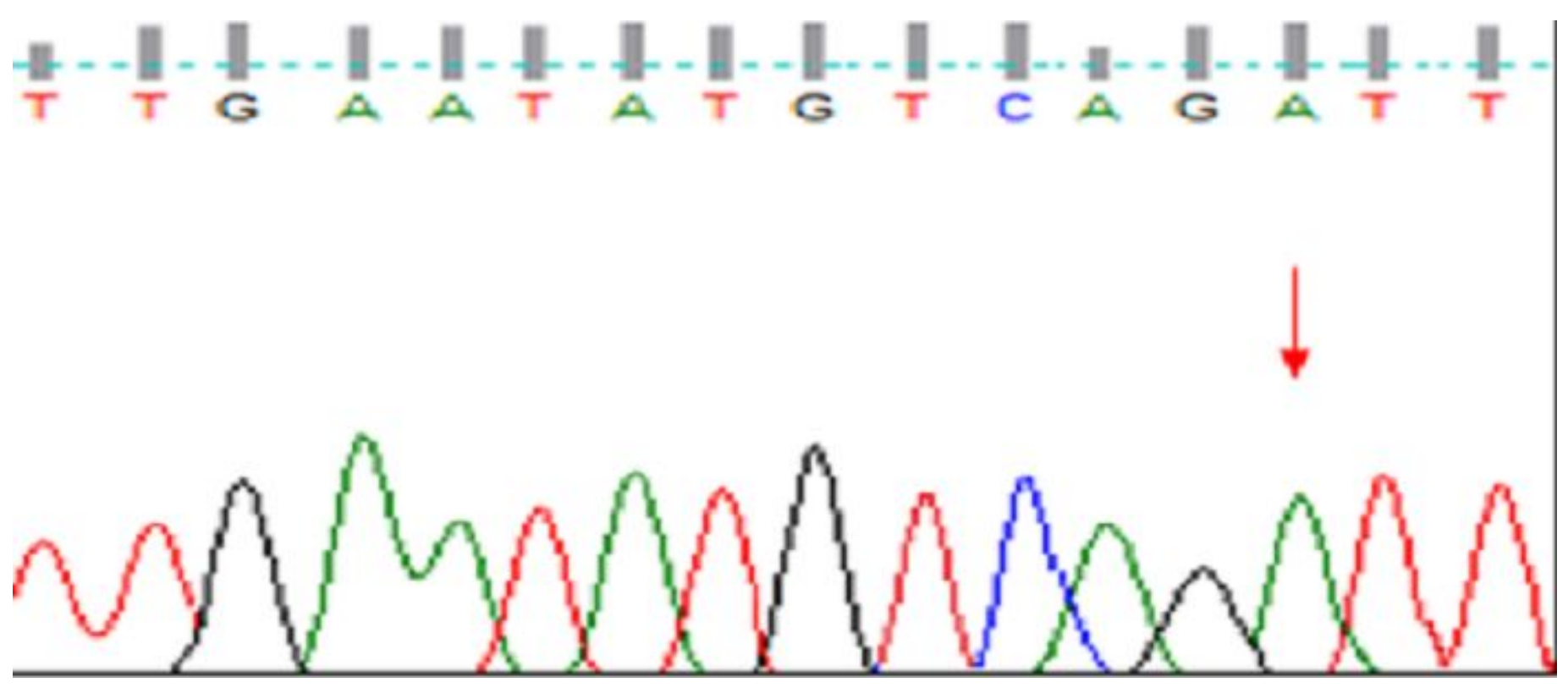

Figure 1

DNA sequencing result from Exon 7 of the HEXB gene showing p.A278V mutation 


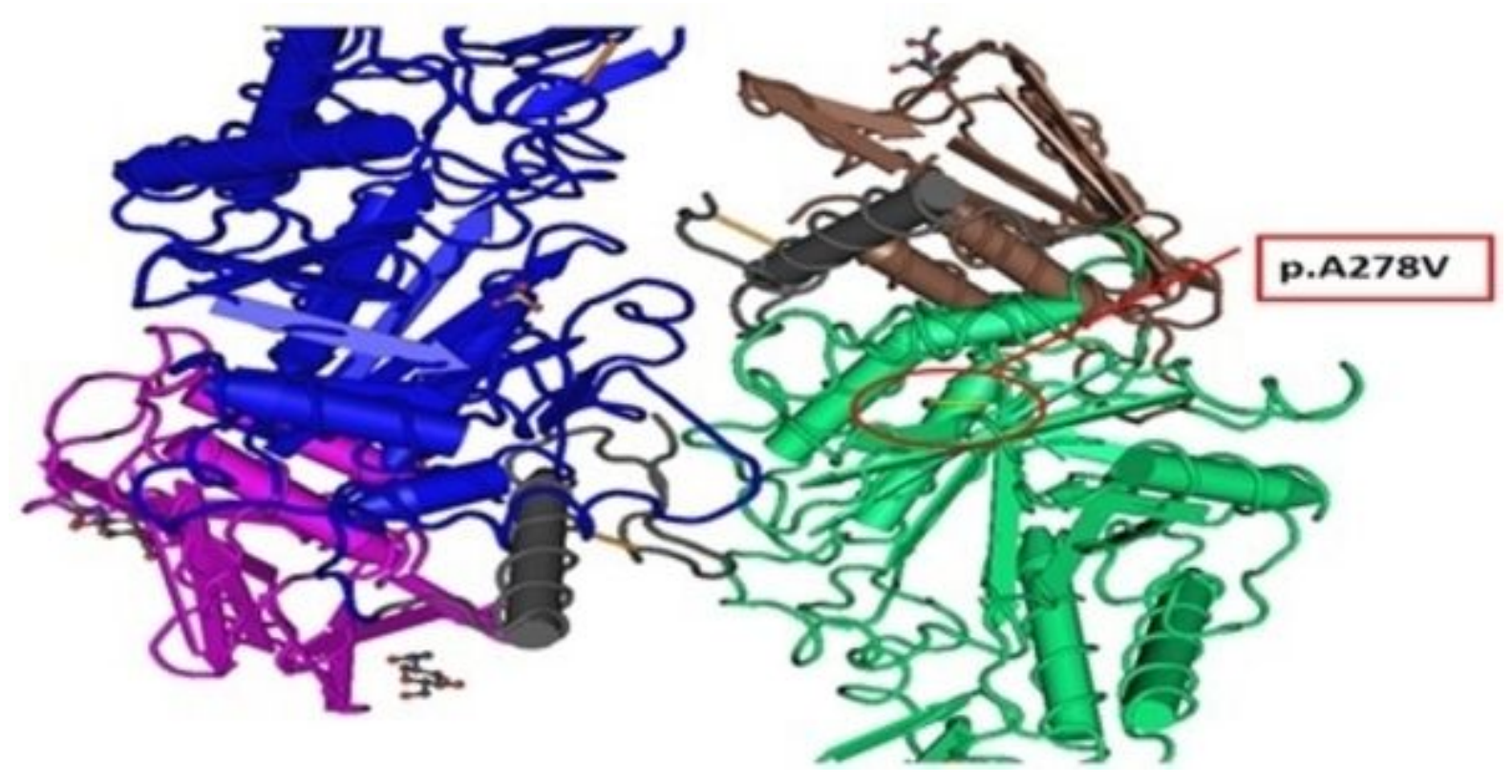

Figure 2

Location of HEXB mutation on the 3-dimensional modeling.

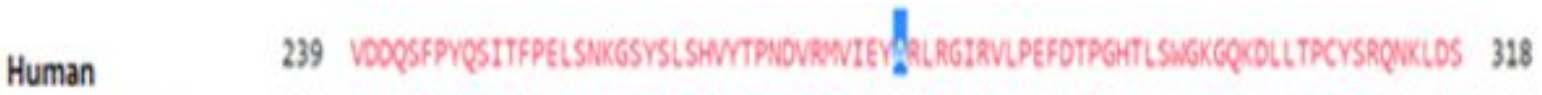

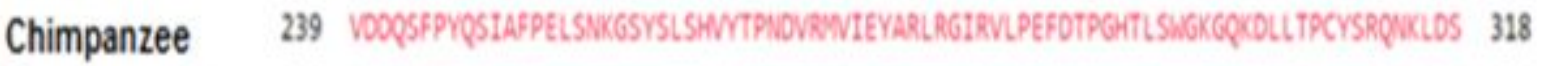

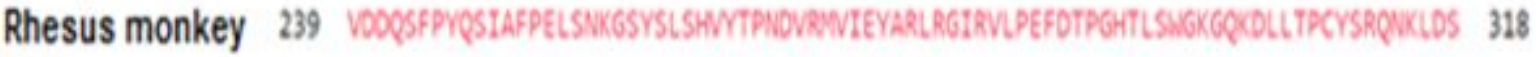

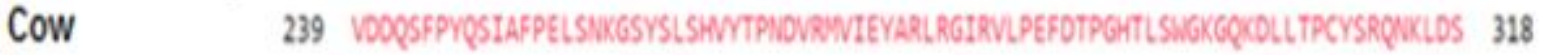

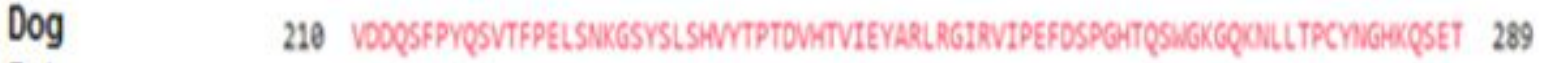

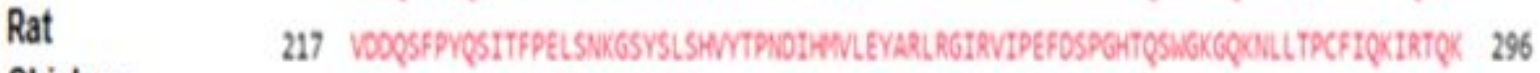

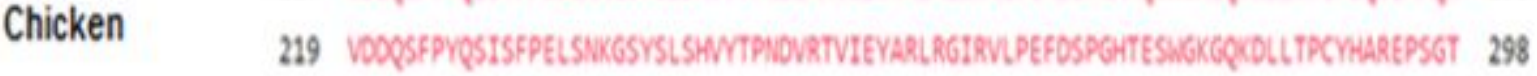

Figure 3

Multiple protein alignment highlighting the evolutionary conservation of Alanin 278 in the human betahexosaminidase subunit beta amino acid sequences. 


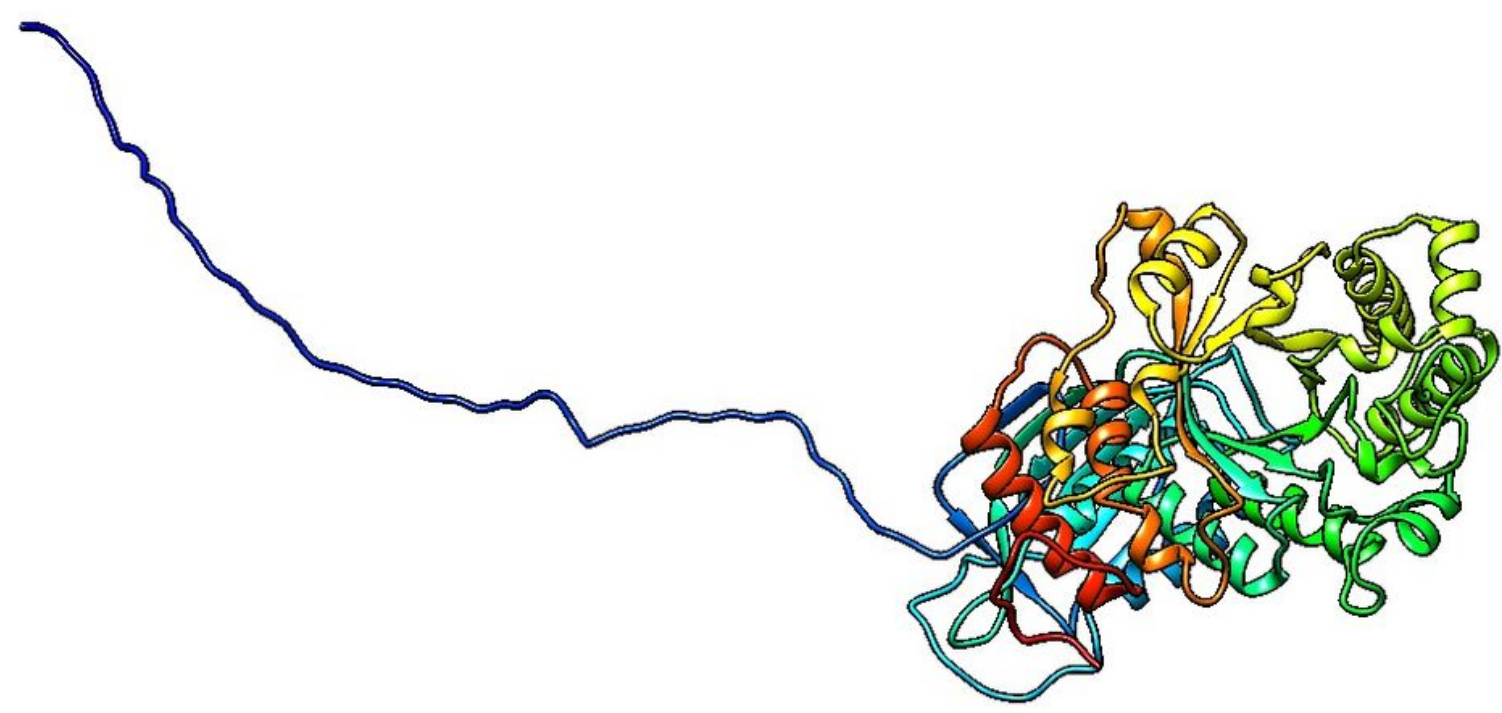

Figure 4

interaction of mutant protein to GDL. 